\title{
Direct measurement and characterization of active photosynthesis zones inside wastewater remediating and biofuel producing microalgal biofilms
}

\section{Authors: Colin Fox \& Albert Parker}

NOTICE: this is the author's version of a work that was accepted for publication in Bioresource Technology. Changes resulting from the publishing process, such as peer review, editing, corrections, structural formatting, and other quality control mechanisms may not be reflected in this document. Changes may have been made to this work since it was submitted for publication. A definitive version was subsequently published in Bioresource Technology, 156, March 2016. DOI\#10.1016/j.biortech.2014.01.001.

Bernstein HC, Kesaano M, Moll K, Smith T, Gerlach R, Carlson RP, Miller CD, Peyton BM, Cooksey KE, Gardner RD, Sims RC, "Direct measurement and characterization of active photosynthesis zones inside wastewater remediating and biofuel producing microalgal biofilms," Bioresource Technology 156: March 2014 206-215. 


\section{Direct measurement and characterization of active photosynthesis zones inside wastewater remediating and biofuel producing microalgal biofilms}

Hans C. Bernstein ${ }^{a, b, c}$, Maureen Kesaano ${ }^{d}$, Karen Moll ${ }^{a, e}$, Terence Smith ${ }^{d}$, Robin Gerlach ${ }^{a, b}$, Ross P. Carlson ${ }^{\mathrm{a}, \mathrm{b}}$, Charles D. Miller ${ }^{\mathrm{d}}$, Brent M. Peyton ${ }^{\mathrm{a}, \mathrm{b}}$, Keith E. Cooksey ${ }^{\mathrm{f}}$, Robert D. Gardner $\mathrm{a}, \mathrm{b}, *$

Ronald C. Sims d,*

a Center for Biofilm Engineering, Montana State University, Bozeman, MT 59717, United States

$\mathrm{b}$ Department of Chemical and Biological Engineering, Montana State University, Bozeman, MT 59717, United States

c Chemical and Biological Signature Science, Pacific Northwest National Laboratories, Richland, WA 99352, United States

d Department of Biological Engineering, Utah State University, Logan, UT 84322, United States

e Department of Microbiology, Montana State University, Bozeman, MT 59717, United States

$\mathrm{f}_{\text {Environmental Biotechnology Consultants, Manhattan, MT 59741, United States }}$

A B S T R A C T

Microalgal biofilm based technologies are of keen interest due to their high biomass concentrations and ability to utilize light and $\mathrm{CO}_{2}$. While photoautotrophic biofilms have long been used for wastewater remediation, biofuel production represents a relatively new and under-represented focus area. However, the direct measurement and characterization of fundamental parameters required for industrial control are challenging due to biofilm heterogeneity. This study evaluated oxygenic photosynthesis and respira-tion on two distinct microalgal biofilms cultured using a novel rotating algal biofilm reactor operated at field- and laboratory-scales. Clear differences in oxygenic photosynthesis and respiration were observed based on different culturing conditions, microalgal composition, light intensity and nitrogen availability. The cultures were also evaluated as potential biofuel synthesis strategies. Nitrogen depletion was not found to have the same effect on lipid accumulation compared to traditional planktonic microalgal stud-ies. Physiological characterizations of these microalgal biofilms identify fundamental parameters needed to understand and control process optimization.

I N T R O D U C T I O N

Photoautotrophic microorganisms are used as biotechnology platforms for many applications including biofuel production,wastewater remediation, carbon sequestration, and agriculture (Christenson and Sims, 2011; Ordog et al., 2004; Pokoo-Aikins et al., 2010). Of these, microalgal biofuel production has been iden-tified as especially promising due to its potential for sustainable supplementation or replacement of fossil fuels (Chisti, 2007; $\mathrm{Hu}$ et al., 2008). Traditionally microalgae biotechnologies have focused on suspended, planktonic, culturing methodologies designed to facilitate photo-production; the capture and conversion of energy from photons into chemical energy stored in extractable biomolecules (e.g., lipids). This study focuses on characterization 
of oxygenic photosynthesis and respiration in photo-biofilm reactors, an alternative and often under-represented growth scenario with benefits over planktonic cultures such as high cell density; which inherently facilitates harvesting and reduces water requirements.

Biofilms are matrix-enclosed microbial cells attached to biological or non-biological surfaces (Hall-Stoodley et al., 2004). Photoautotrophic biofilms, composed of microalgae and/or cyanobacteria, are ubiquitous to nearly all photic aquatic environments. An important attribute of biofilms is that they both create and are functionally controlled by gradients in substrates, products and energy sources (Stewart and Franklin, 2008). Spatial gradients in light have been shown to directly control rates of oxygenic photosynthesis and corresponding oxygen concentrations inside biofilms (Kuhl et al., 1996). Oxygen gradients in biofilms are directly influenced by diffusion rates and can result in localized supersaturated concentrations (with respect to air oxygen concentrations) during active oxygenic photosynthesis. The resulting high oxygen concentrations can inhibit $\mathrm{CO}_{2}$ fixation by competing as a substrate for ribulose 1,5-bisphosphate carboxylase-oxygenase (RuBisCO) (Falkowski and Raven, 1997; Glud et al., 1992; Kliphuis et al., 2011). This competition subsequently limits photo-production of carbon storage compounds. Thus, the characterization of spatial gradients in oxygenic photosynthesis and respiration activities is a key consideration for microalgal biofilm-based technologies.

This study employed a recently developed rotating algal biofilm reactor (RABR) that was designed, built and tested at both the laboratory scale (lab-RABR) and pilot field scale (field-RABR) (Christenson and Sims, 2012) (Fig. 1). The advantage of the RABR is the ability to simultaneously facilitate algal growth and dewatering while achieving high biomass concentration. Biofilm reactors can also reduce the water and energy requirements for biomass and photo-production compared to traditional suspended culturing strategies (Ozkan et al., 2012). The RABR and other algal-based biofilm technologies have been investigated for their potential to concurrently remediate wastewater and produce biofuel precursor molecules (Boelee et al., 2012; Farooq et al., 2013). The RABR can facilitate efficient biomass harvesting via the reported spool harvesting technique (Christenson and Sims, 2012). However, optimal biomass harvesting practices need to be determined in the context of biofilm specific physiology, such as optimal biomass areal density and biofilm thickness as it relates to active photo-production and photosynthesis zones.

The current study focuses on spatial physiological characterization of microalgal biofilms cultured through the RABR method. The specific aims of this study were to:

1) characterize and compare two different RABR biofilms (wastewater remediating and potentially biodiesel producing) in the context of active photosynthesis zones by directly measuring spatial gradients in oxygen and photosynthesis microprofiles, as well as, determining rates of photosynthesis and respiration.

2) Characterize and compare the biofuel potential and (neutral lipid) precursor biomolecule composition in these biofilms. In addition to specific aim 2, nitrate starvation was investigated as a potential strategy for inducing lipid accumulation in the lab-scale RABR biofilms.

\section{Methods}

\subsection{Laboratory strains, culturing conditions, and biomass sampling}

The Chlorophyte isolate Botryococcus sp. strain WC-2B (hereinafter referred to as WC-2B) was cultured with $8 \mathrm{~L}$ lab-RABRs operated in batch mode. WC-2B was isolated from an alkaline stream in Yellowstone National Park (USA), confirmed unialgal using SSU 18S rDNA and revealed 99\% alignment with Botryococcus sedeticus UTEX 2629, which has previously been described (Senousy et al., 2004). Reactors were operated in triplicate and grown at $25^{\circ} \mathrm{C}$ in Bold's basal medium buffered with $25 \mathrm{mM}$ 2-[N-cyclohexylamino]-ethane-sulfonic acid (CHES, pKa 9.3) and rotated at 15.3 RPM. All RABR experiments were loaded with untreated cotton cord as the biofilm-substratum $(0.64 \mathrm{~cm}$ diameter) (Christenson and Sims, 2012). The lab-RABRs consisted of cords coiled onto plastic cylindrical-spools ( $10 \mathrm{~cm}$ diameter) submerged approximately $5 \mathrm{~cm}$ in the liquid medium. The lab-RABRs were cultured under custom light emitting diode (LED) banks (Box Elder Innovations, LLC and T\&L Design, Box Elder UT) programmed with LabVIEW (National Instruments Corp.) to simulate a diurnal cycle with photosynthetically active radiation (PAR) values ranging from 0 to $900 \mu \mathrm{mol}$ photons $\mathrm{m}^{-2} \mathrm{~s}^{-1}$ on a 14:10 L/D diel cycle following Eq. (1).

$I=\cos \left(\frac{\pi}{t_{L}} *\left(t-t_{M}\right)\right)^{2}$

where $I$ is the light intensity, $t_{L}$ is the total light time in minutes, $t$ is the independent time variable, and $t_{M}$ is the midpoint time corresponding to the maximum light intensity.

Nitrate concentrations in the medium were monitored using NitraVer 5 pillow packets $(\mathrm{HACH})$. Concentrated medium $(10 \times)$ and supplemental $\mathrm{diH}_{2} \mathrm{O}$ (deionized) were added, as needed, to maintain nutrient replete conditions and offset evaporation. Culturing and sampling were performed under non-aseptic conditions (i.e., open-air). Nitrate depletion was induced (after 28 days of replete culturing) by removing all liquid medium from the reactors followed by immediate replacement with Bold's basal medium without nitrate. Analysis of the biofilms after nitrate depletion was performed $60 \mathrm{~h}$ post depletion.

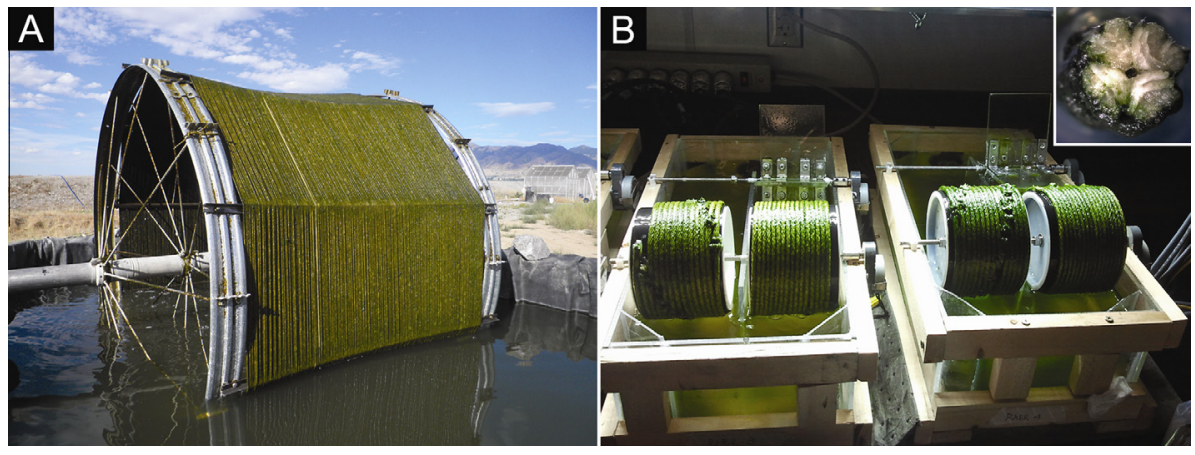

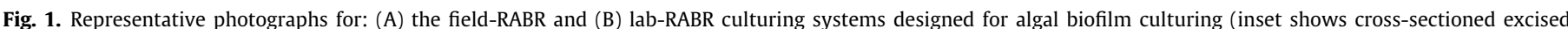

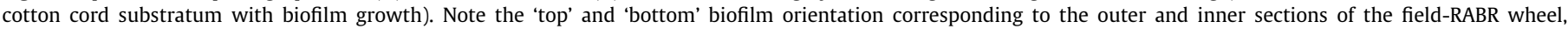
respectively. 
Biomass cell dry weights (CDW, $\mathrm{g}_{\mathrm{CDW}} \mathrm{cm}^{-2}$ ) were obtained throughout culturing by excising a known length of cotton cord and its attached biofilm, followed by biofilm removal into preweighed aluminum weigh boats. The biomass was dried at $70^{\circ} \mathrm{C}$ for $18 \mathrm{~h}$ until the biomass weight was constant. Biomass CDWs were calculated by subtracting the dry weight of the pre-weighed aluminum boat from the oven dried boat with biomass and normalizing by the cylindrical surface area for the length of cotton cord substratum excised.

\subsection{Outdoor culturing conditions}

Field scale biofilms were cultured outdoors (August 10thOctober 17th 2012, Logan, UT, USA) with a pilot scale RABR (field-RABR) unit constructed in accordance with previously described methods (Christenson and Sims, 2012). Briefly, biofilms were grown on cotton cord (identical to lab-RABR experiments) coiled onto aluminum wheels (193 cm in diameter) which rotated (1.25 RPM) partially submerged in approximately $14,000 \mathrm{~L}$ tanks (approximately 10,700 L liquid volume). An important difference from the lab-RABR was that the cord-substratum of the field-RABR was exposed to light and nutrients from top and bottom (discussed further below). The field-RABR was placed in a continuous flow channel of wastewater $\left(18.9^{\circ} \mathrm{C}\right.$ and $\mathrm{pH}$ 7.4) fed at approximately $1.25 \mathrm{~L} \mathrm{~min}^{-1}$, which was drawn from the final pond of the outdoor wastewater lagoon facility (Logan, UT, USA).

\subsection{Oxygen microsensor analysis}

Microsensor measurements were performed using Clark-type oxygen micro-electrodes with outside tip diameters of $25 \mu \mathrm{m}$, response time $<5 \mathrm{~s}$ and $<5 \%$ stirring sensitivity (Unisense, $\mathrm{A} / \mathrm{S}$ ) (Revsbech, 1989). Amplification and sensor positioning were controlled with a microsensor multi-meter coupled with an ADC216 USB converter and a motor controlled micromanipulator. Data collection was aided by software packages, SensorTrace Pro ver. 3.0.1 and Sloper ver. 3.0.3 (Unisense, A/S). Two point calibrations were performed in air-saturated $\mathrm{diH}_{2} \mathrm{O}\left(\left[\mathrm{O}_{2}\right] \approx 260 \mu \mathrm{M}\right)$ and in a $1 \mathrm{M} \mathrm{NaOH}, 0.1 \mathrm{M}$ ascorbic acid solution (anoxic standard). Calibrations were repeatedly checked in the anoxic standard and in air-saturated $\mathrm{diH}_{2} \mathrm{O}$ throughout the experiments. Microsensor measurements were performed between 21 and $25{ }^{\circ} \mathrm{C}$ under both dark and light conditions ( $P A R=700 \mu \mathrm{mol}$ photons $\mathrm{m}^{-2} \mathrm{~s}^{-1}$ ). Spatially resolved $\mathrm{O}_{2}$ measurements were performed in onedimension (depth-wise) from the biofilm-air interface down towards the cotton cord substratum in 25-100 $\mu \mathrm{m}$ steps. The effective diffusion coefficient $\left(D_{e}\right)$ for $\mathrm{O}_{2}$ in the microalgal biofilms was estimated to be $1.2 \times 10^{-5} \mathrm{~cm}^{2} \mathrm{~s}^{-1}$, by assuming it to be $50 \%$ of the aqueous value corresponding to water at $25^{\circ} \mathrm{C}$ (Stewart, 1998). The oxygen micro-profile and light:dark shift techniques used here have been previously described in detail (Bernstein et al., 2013; Glud et al., 1992; Kuhl et al., 1996; Lassen et al., 1998). Briefly, Fick's law was used to calculate the total oxygen flux exported from the surface of the biofilm (net areal rate of biofilm photosynthesis or $P_{n}$ ) and from the photic zone inside the biofilm (net areal rate of photosynthesis of the photic zone or $\left.P_{n, p h o t}\right)$. Additionally, the light:dark shift measurements were used to estimate gross photosynthesis profiles and areal rates $\left(P_{g}\right)$ which represent the total amount of oxygenic photosynthesis under the assumptions that: (i) there is an initial steady-state $\mathrm{O}_{2}$ distribution prior to darkening, (ii) the $\mathrm{O}_{2}$ consumption rate is identical between the light and dark time periods, (iii) the $\mathrm{O}_{2}$ diffusion coefficient remains constant during the measurement time at each position. Detailed calculations for oxygen transport, photosynthesis, photosynthesis-coupled respiration and dark-respiration processes are included in the Supplementary Section for this manuscript.

\subsection{Lipid analysis}

At the time of oxygen microsensor analysis, bulk biomass was harvested from the RABRs and washed four times by centrifugation $(5000 \times g)$ and resuspended in $\mathrm{diH}_{2} \mathrm{O}$ to remove medium salts. After washing, the biomass was centrifuged and the pelleted biomass was frozen for lyophilization and lipid analysis. Analysis of free fatty acids, mono-, di-, and tri-acyl glycerols (FFA, MAG, DAG, and TAG, respectively) was performed in accordance to the bead beating extraction method coupled with gas chromatographyflame ionization detection (GC-FID) reported by Lohman et al (2013). Additionally, biofuel potential, defined as total fatty acid methyl esters (FAME) produced directly from the biomass (Eustance et al., 2013; Gardner et al., 2013), along with FAME profiles were determined by a previously described method of direct in situ biomass transesterification using gas chromatography-mass spectroscopy (GC-MS) (Lohman et al., 2013).

\section{Results and discussion}

\subsection{Biofilm cultivation}

Biofilms were cultured on cotton cord substratum during fieldand laboratory-scale RABR experiments (Fig. 1). Samples from the lab-RABR were analyzed based on nitrate replete or deplete conditions. Samples from the field-RABR were separated according to growth orientation on the substratum. The field-RABR 'top' and 'bottom' samples correspond to biofilms formed on the outer and inner section of the rotating wheel, respectively. The field-RABR top biofilms were cultured in an orientation directly exposed to ambient sun light (average daily maximum PAR $=1715 \mu \mathrm{mol}$ photons $\mathrm{m}^{-2} \mathrm{~s}^{-1}$ ) compared to the more shaded bottom biofilms (average daily maximum $\mathrm{PAR}=231 \mu \mathrm{mol}$ photons $\mathrm{m}^{-2} \mathrm{~s}^{-1}$ ). Hence, there were four chosen biofilm sample-types analyzed and compared in this study: (i) lab-RABR biofilm that is nitrate replete, (ii) lab-RABR biofilm that is nitrate deplete ( $60 \mathrm{~h}$ deplete culturing), (iii) field-RABR biofilm cultured on the top (outer wheel biofilm), and (iv) field-RABR biofilm cultured on the bottom (inner wheel biofilm). It is important to emphasize that the laboratoryand field-RABR systems are not identical, represent two different process objectives and are intended to be compared independently of each other. However, a future goal for the RABR technology is to better integrate the wastewater remediating and biofuel producing processes; hence a minimal number of comparisons based on general biofilm physiology are made between the two systems.

The maximum specific growth rates, measured during exponential phase, were 0.09 and 0.17 day $^{-1}$ for the laboratory and field cultured biofilms, respectively. The maximum measured biomass areal density (observed during stationary phase) were 0.36 and $0.65 \mathrm{~g}_{\mathrm{CDW}} \mathrm{cm}^{-2}$ for the lab- and field-RABRs, respectively. The final biomass areal density decreased by $0.01 \mathrm{~g}_{\mathrm{CDW}} \mathrm{cm}^{-2} 60 \mathrm{~h}$ post nitrate depletion in the lab-RABR biofilms, potentially indicating minor biomass sloughing or degradation. The measured biofilm thickness (distance from substratum to biofilm surface at late stationary phase) was approximately $1 \mathrm{~mm}$ for each lab-RABR biofilm (nitrate replete or deplete) and approximately $2 \mathrm{~mm}$ for each fieldRABR biofilm (top and bottom).

\subsection{Field-RABR for wastewater remediation}

\subsubsection{Biofilm heterogeneity}

Direct, spatially resolved measurements of steady-state oxygen profiles revealed differences between the biofilms formed on the top and bottom of the field-RABR wastewater remediating system. The illuminated portions of both biofilms near the surfaces became 
supersaturated with $\mathrm{O}_{2}$, reaching concentrations over $600 \mu \mathrm{M}$ which was approximately $3 \mathrm{X}$ the measured $\mathrm{O}_{2}$ concentration of the bulk wastewater (Fig. 2A and B). Both biofilms were oxic to depths of approximately $1800 \mu \mathrm{m}$ beneath the biofilm surface while illuminated (Table 1 ). Steady-state oxygen profiles were also obtained after 15 min of dark conditioning (Fig. 3C and B) and the corresponding oxic-zone depths were 700 and $450 \mu \mathrm{m}$ in the top and bottom biofilms, respectively. This is evidence for higher oxygen consumption potential in the less-illuminated (bottom) biofilm on the inside of the spools (discussed in more detail below).

Oxygen gradients measured in the steady-state microprofiles show that these wastewater remediating biofilms maintain spatially varied microenvironments which may promote niche environments capable of supporting different microbial physiologies.
A significant portion of both biofilms (top and bottom) remained anoxic during constant illumination at $700 \mu \mathrm{mol}$ photons $\mathrm{m}^{-2} \mathrm{~s}^{-1}$ (approximately 10\%) and in the dark (approximately 50\%). However, it is possible that these biofilms become fully oxic at or near peak solar irradiance during field cultivation. In the field, these systems are also subject to temporal gradients in solar irradiance, temperature and nutrient flux. It is important to note that the measurements reported in this study are specific for standardized and constant incident irradiance and only represent comparative physiological potentials for these biofilms.

The field-RABR was inoculated with the native wastewater microbial community and was composed of a complex mixture of environmental biofilm-forming microorganisms including phototrophs and heterotrophs. Initial 454 pyrosequence analyses indicated a high level of diversity in the field-RABR biofilms, where
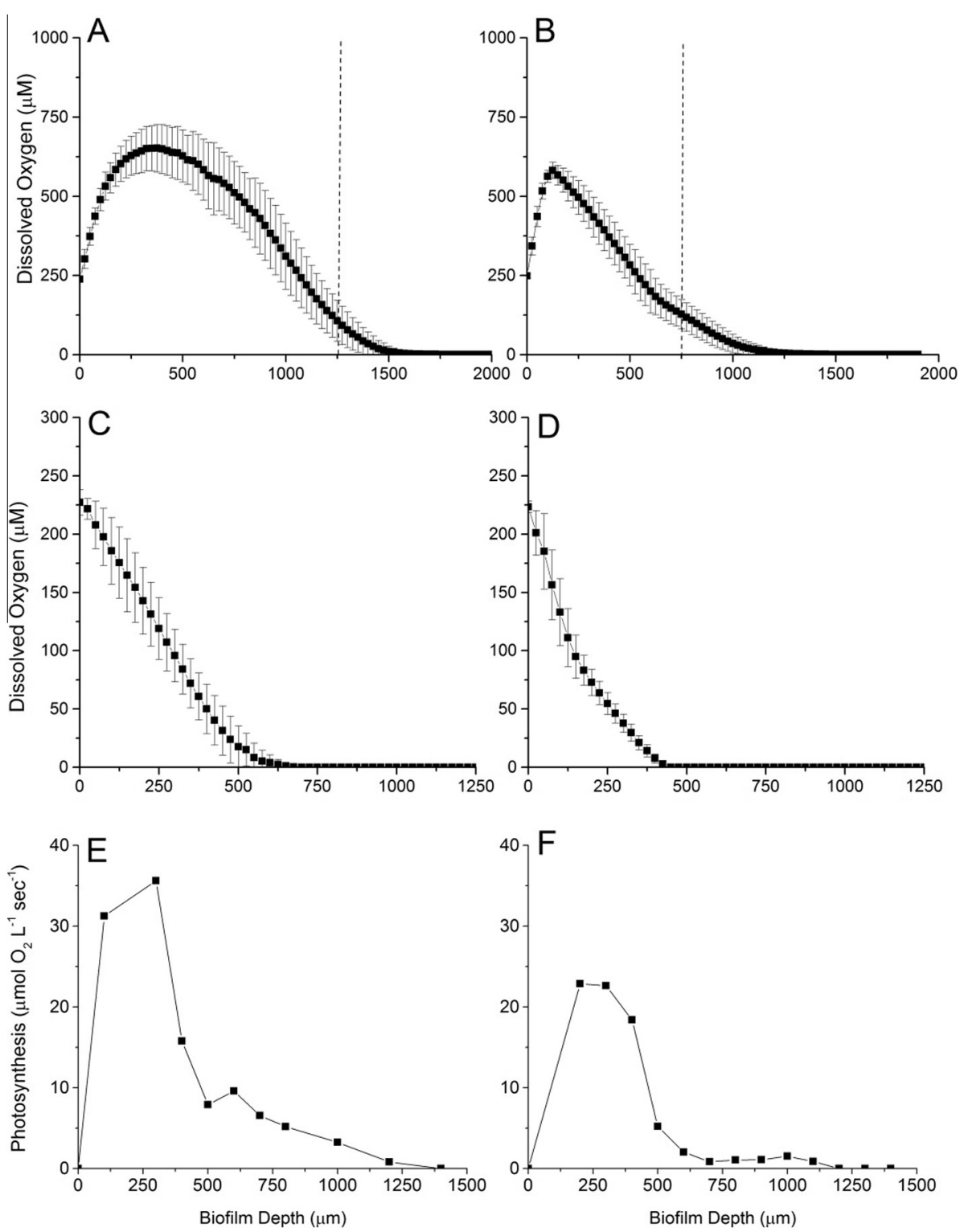

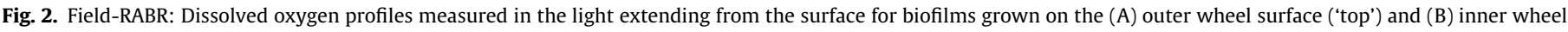

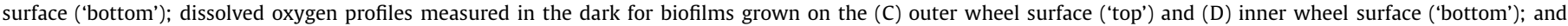

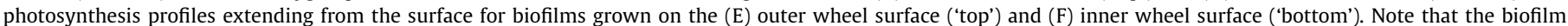

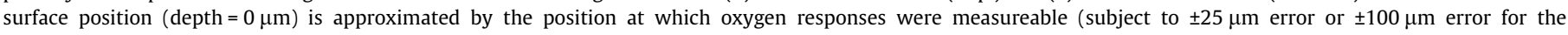

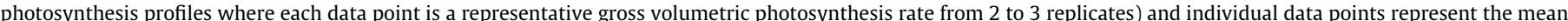

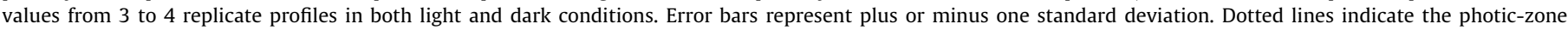
termination depth, estimated from the light:dark shift method. Note the scale change on the $x$-axis. 
Table 1

Measurements of areal photosynthesis rates, areal respiration rates and relevant depth scales for the laboratory- and field-RABR cultured biofilms.

\begin{tabular}{|c|c|c|c|c|}
\hline Areal rates $\left(\mu \mathrm{mol} \mathrm{O} \mathrm{Cm}^{-2} \mathrm{~s}^{-1}\right)$ & $\begin{array}{l}\text { Field RABR top } \\
\text { biofilm }\end{array}$ & $\begin{array}{l}\text { Field RABR bottom } \\
\text { biofilm }\end{array}$ & $\begin{array}{l}\text { Laboratory RABR nitrate } \\
\text { replete }\end{array}$ & $\begin{array}{l}\text { Laboratory RABR nitrate } \\
\text { deplete }\end{array}$ \\
\hline Photosynthesis, $P_{g}$ & $11.84 \times 10^{-4 a}$ & $5.23 \times 10^{-4 a}$ & $7.51 \times 10^{-4 a}$ & $5.70 \times 10^{-4 a}$ \\
\hline Net areal rate of biofilm photosynthesis, $P_{n}\left(\% P_{g}\right)$ & $3.01 \times 10^{-4}(25.4 \%)$ & $3.55 \times 10^{-4}(67.9 \%)$ & $2.31 \times 10^{-4}(30.8 \%)$ & $2.41 \times 10^{-4}(42.3 \%)$ \\
\hline $\begin{array}{l}\text { Net areal rate of photic zone photosynthesis, } P_{n, p h o t} \text {, } \\
\left(\% P_{g}\right)\end{array}$ & $3.64 \times 10^{-4}(30.7 \%)$ & $3.96 \times 10^{-4}(75.7 \%)$ & $3.10 \times 10^{-4}(41.3 \%)$ & $2.91 \times 10^{-4}(51.1 \%)$ \\
\hline Areal respiration of the biofilm, $R_{\text {light }}\left(\% P_{g}\right)$ & $8.83 \times 10^{-4}(74.6 \%)$ & $1.68 \times 10^{-4}(32.1 \%)$ & $5.20 \times 10^{-4}(69.2 \%)$ & $3.29 \times 10^{-4}(57.7 \%)$ \\
\hline Areal respiration of the photic zone, $R_{\text {phot }}\left(\% P_{g}\right)$ & $8.20 \times 10^{-4}(69.3 \%)$ & $1.27 \times 10^{-4}(24.3 \%)$ & $4.41 \cdot 10^{-4}(58.7 \%)$ & $2.79 \times 10^{-4}(48.9 \%)$ \\
\hline Respiration in the dark, $R_{\text {dark }}$ & $0.54 \times 10^{-4}$ & $1.11 \times 10^{-4}$ & $0.65 \times 10^{-4}$ & $0.74 \times 10^{-4}$ \\
\hline Depth of photic zone, $L_{\text {phot }}(\mu \mathrm{m})$ & $1100 \pm 200^{\mathrm{b}}$ & $900 \pm 200^{\mathrm{b}}$ & $675 \pm 25^{\mathrm{b}}$ & $650 \pm 25^{b}$ \\
\hline Depth of oxic zone in light $(\mu \mathrm{m})$ & $1750 \pm 25^{\mathrm{b}}$ & $1800 \pm 25^{\mathrm{b}}$ & $>2675$ & $>2675$ \\
\hline Depth of oxic zone in dark $(\mu \mathrm{m})$ & $700 \pm 25^{\mathrm{b}}$ & $450 \pm 25^{\mathrm{b}}$ & $850 \pm 25^{b}$ & $1150 \pm 25^{\mathrm{b}}$ \\
\hline
\end{tabular}

a Mean of 2-3 independent measurements plus or minus a range of $25 \%$ from the mean.

b Plus or minus measurement step-size, $n=2-3$.

cyanobacteria (predominately Oscillatoria sp. and Leptolyngbya sp.) and bacterial heterotrophs accounted for significant fractions of the microbial population. However, further molecular work is required to elucidate the microbial community differences between the two biofilms with respect to their orientation of growth. It is important to reemphasize that the 'top' and 'bottom' biofilms were formed simultaneously on different sides of the same cotton cord substratum and analyzed with microsensors ex situ under identical conditions. Other than growth orientation, these biofilms were cultured identically and were only spatially separated by the diameter of the cotton cord substratum $(0.64 \mathrm{~cm})$.

\subsubsection{Oxygenic photosynthesis}

Direct measurements of oxygenic photosynthesis rates quantified fundamental physiological differences in the field-RABR biofilms based only on orientation of biofilm formation (Fig. $2 \mathrm{E}$ and F). The measured areal rate of gross photosynthesis $\left(P_{g}\right)$ in the top biofilm was approximately $2 \times$ greater than the bottom, signifying a much higher potential for photo-productivity (proportional to $P_{g}$ ) (Table 1 ). This result was attributed to the availability of solar irradiance (PAR) during biofilm growth/formation which differed between 1715 and $231 \mu \mathrm{mol}$ photons $\mathrm{m}^{-2} \mathrm{~s}^{-1}$ for the top and bottom biofilms, respectively.

The active zone of photosynthesis is defined here as the position in the biofilm where the volumetric gross photosynthesis rate $\left[P_{g}(z)\right]$ is greater than zero and its depth is assumed to be equal to the biofilm photic zone ( $L_{\mathrm{phot}}$ ). The $L_{\mathrm{phot}}$ value was only slightly higher in the top biofilm (Table 1) indicating that the penetration depths of actinic light are comparable when illuminated at the same incident irradiance. The minor differences observed in $L_{\mathrm{phot}}$ values may translate into minor differences in biofilm material properties which may influence the oxygen effective diffusion coefficient, as $L_{\mathrm{phot}}$ is resolved based on photogenic oxygen changes; however, these variances are expected to be very small based on previously reported measurements (Stewart, 1998) and were not considered here in detail. A key observation for this system is that the top oriented biofilms are capable of producing oxygen at greater than twice the rate per photon attenuated than the neighboring bottom biofilm while the respective zones of active photosynthesis are nearly identical under standardized incident irradiance. This observation qualitatively indicates that the areal quantum yields are greater for the biofilms formed under a higher incident solar irradiance. Rigorous quantification of spatially defined quantum yields and photosynthetic efficiencies are beyond the scope of this study although the present results are consistent with established photo-physiological observations (Falkowski and Raven, 1997).

Net areal photosynthesis rates were equated to the diffusive flux of oxygen transported from the biofilm surface $\left(P_{n}\right)$ or the photic zone $\left(P_{n, p h o t}\right)$, and both measurements were greater in the bottom formed biofilms compared to the top oriented samples (Table 1). This difference is more pronounced and meaningful when interpreted as a percentage of $P_{g}$ which is a proxy for the total photosynthetically derived oxygen. Net photosynthesis rates for the entire biofilm $\left(P_{n}\right)$ represent $67.9 \%$ of $P_{g}$ in the bottom biofilm as compared to only $25.4 \%$ in the top. These percentage differences are even greater when evaluated for $P_{n, p h o t}$, which includes consideration of oxygen transported to the anoxic portions in the biofilm. These results confirm that net oxygen production rates alone are not representative of the oxygenic photosynthesis potential for these samples and that the bottom biofilms have the capacity to provide a greater flux of oxygen to bulk wastewater environment.

The $P_{n}$ values measured for this study are only representative of steady-state reaction and diffusion processes. However, the rotating mechanism employed by the RABR alternates the biofilms between different light and fluid regimes in a periodic fashion corresponding to the submerged-liquid and ambient air surroundings. Diffusive oxygen flux was measured inside the biofilms and the steady-state oxygen profiles obtained on biofilms exposed to ambient air did not provide enough resolution to identify or determine the thicknesses of the diffusive boundary layer (DBL) at the surface of the biofilms. However, DBLs almost certainly were present and are not ruled out as important regulating factors in the oxygen transport processes, especially while being exposed to the liquid medium during rotation. It has been previously established that DBL thickness is a function of the velocity differential between the biofilm and bulk fluid (Jorgensen and Marais, 1990; Kuhl et al., 1996). This is an important consideration for RABR operation since the rotational speed can be optimized to reduce the effects of mass transfer limitations external to the biofilm. This highlights a future area of characterization for the field-RABR biofilms that has the potential to enhance photosynthetic biofilm productivity by minimizing mass transfer limitations.

\subsubsection{Areal respiration rates}

The difference between gross and net areal photosynthesis rates provided direct measurements of photosynthesis-coupled respiration and revealed physiological distinctions between the two fieldRABR biofilms. Areal photosynthesis coupled respiration rates were measured during illumination for the entire biofilm $\left(R_{\text {light }}\right)$ and within just the photic zone $\left(R_{\text {phot }}\right)$. Both measurements were more than $5 \times$ higher in the top biofilms compared to the bottom (Table 1). Respiration rates accounted for greater percentages of $P_{g}$ than the corresponding $P_{n}$ values in the top biofilms. The opposite was true for the bottom biofilms.

In contrast to the photosynthesis-coupled respiration rates, areal respiration rates in the dark $\left(R_{\text {dark }}\right)$ were approximately 

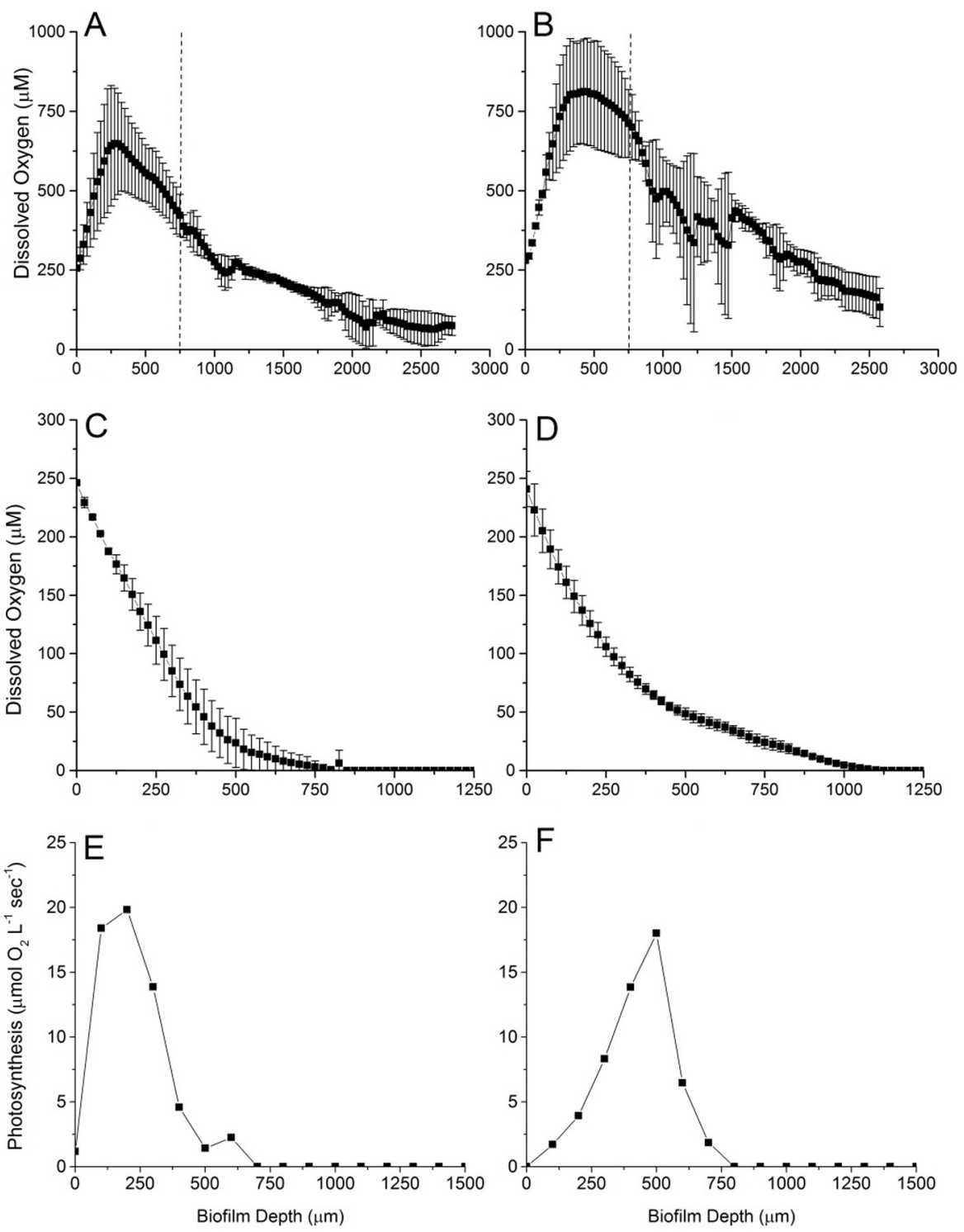

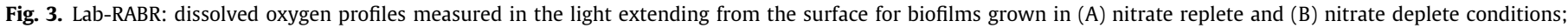

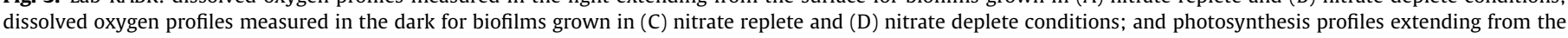

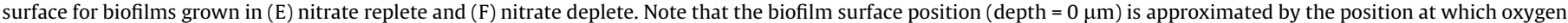

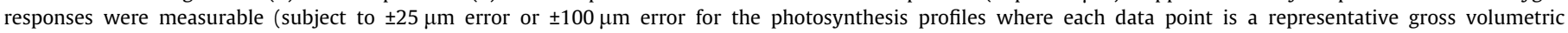

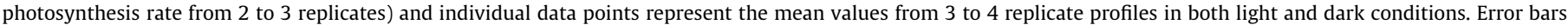

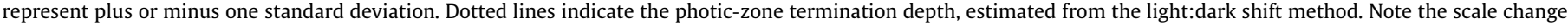
on the $x$-axis.

$2 \times$ greater for the bottom biofilms compared to the top biofilms (Table 1). Respiration rates corresponded directly to higher localized oxygen concentrations. This observation indicates that respiration in these biofilm consortia increases with oxygen concentration and production rate which are both functions of actinic light availability. This provides evidence of photo-respiration processes acting in concert with heterotrophic oxygen consumption. The bottom biofilms appear to have a higher capacity for light-independent heterotrophic respiration compared to the top biofilms which is evidenced by the higher $R_{\text {dark }}$ values.

Photosynthesis-coupled respiration is defined here to include any respiration occurring in the active zone of photosynthesis and can be advantageous to overall photo-production by lowering the localized $\mathrm{O}_{2} / \mathrm{CO}_{2}$ ratio inside the biofilm and resulting in higher selectivity for $\mathrm{CO}_{2}$ fixation at the RuBisCO complex (Falkowski and Raven, 1997; Glud et al., 1992; Kliphuis et al., 2011). Oxygen removal via heterotrophic or non-oxygenic community member activity is hypothesized to be a beneficial attribute to these wastewater remediating biofilm ecosystems. Hence, the encouragement and control of localized respiration processes, independent of photo-respiration, is identified here as a potentially important design feature for RABR operation and other photosynthetic biofilm reactor technologies and should be considered for future optimization of photo-production.

The top oriented field-RABR biofilm samples showed the highest rates of gross-oxygenic photosynthesis and respiration (both $R_{\text {light }}$ and $R_{\text {phot }}$ ). These two processes are tightly coupled inside biofilms and cannot be considered independent from each other. In fact, it has been shown previously that photosynthesis and respiration increase concurrently with increasing irradiance in tightly controlled laboratory cultured algal biofilms (Jensen and Revsbech, 1989). The differences in the photosynthesis and respiration capacities for these biofilms might be the result of the differences in exposure to solar irradiance during the culturing process. The top 
oriented biofilms were formed with a 7.4-fold higher incident irradiance (PAR) compared to the bottom of the cotton cord substratum. This is a practical result since it is well established that different growth environments with respect to solar irradiance availability have been shown to promote different expression levels of components comprising the light harvesting complexes, nonphotosynthetic accessory pigments (e.g., carotenoids) and respiration components (e.g., terminal oxidases) in photosynthetic systems (Falkowski and Raven, 1997).

\subsection{Nitrogen depletion in lab-RABR samples}

\subsubsection{Biofilm heterogeneity}

The lab-RABR biofilms, formed from the known lipid accumulating strain $\mathrm{WC}-2 \mathrm{~B}$, established oxygen gradients under both illuminated and dark conditions. The microprofiles revealed only subtle differences between biofilms subjected to nitrate replete and deplete conditions. Similar to the field-RABR biofilms, the illuminated surface associated positions from both replete and deplete biofilm samples became supersaturated with $\mathrm{O}_{2}$, reaching approximately $3 \times$ the measured $\mathrm{O}_{2}$ concentration of the medium (Fig. 3A and B). During illumination, the oxic zone extended to depths greater than $2675 \mu \mathrm{m}$ below the biofilm surface (approximately $1675 \mu \mathrm{m}$ into the substratum) where the flux of oxygen became very low. The WC-2B biofilms showed oxygen transport, driven by consumption, in portions of the substratum indicating that some biofilm was formed within the cotton cord pore volume. This was also observed by confocal scanning laser microscopy (Supplemental Figs. S2 and S3). These lab-RABR biofilms showed a higher degree of spatial heterogeneity with respect to replicate oxygen profiles compared to the field-RABR biofilms (evident by the larger standard deviations in Fig. 3 as compared to Fig. 2). This increased variance between measurements taken below $L_{\text {phot }}$ positions could result from biofilm spatial heterogeneity specific for cells attached within the cotton material. Steady-state oxygen profiles were also obtained after $15 \mathrm{~min}$ of dark conditioning (Fig. $3 \mathrm{C}$ and $\mathrm{D}$ ). The oxic zones in the absence of light ranged from 850 to 1150 for the nitrate replete and deplete biofilms, respectively; indicating that the nitrogen starved biofilms had a lower potential for heterotrophic oxygen consumption (discussed in more detail below).

\subsubsection{Oxygenic photosynthesis and respiration}

Direct measurements of oxygenic photosynthesis and respiration rates indicated physiological differences in the RABR grown WC-2B biofilms cultured under nitrate replete and deplete conditions (Fig. 3E and F). Again, photosynthesis rates were measured as both net and gross production of photo-chemically derived oxygen at the biofilm scale. The WC-2B biofilms exhibited higher $P_{g}$ values (approximately 30\%) during nitrate replete conditions indicating a greater potential for photo-productivity when not starved for nitrogen (Table 1). The active zones of photosynthesis,

Table 2

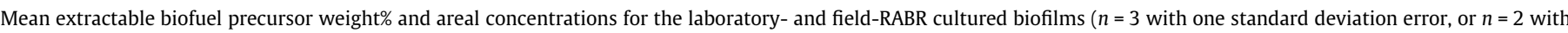
range reported as error).

\begin{tabular}{|c|c|c|c|c|c|c|}
\hline & Condition & Free fatty acid & Mono-acylglycerol & Di-acylglycerol & Tri-acylglycerol & Total extractable \\
\hline \multirow[t]{3}{*}{ Weight\% (w/w) } & LR-replete & $2.24 \pm 0.39$ & $1.17 \pm 0.21$ & $0.76 \pm 0.35$ & $0.11 \pm 0.09$ & $4.27 \pm 0.37$ \\
\hline & LR-deplete & $2.91 \pm 0.46$ & $1.48 \pm 0.21$ & $1.98 \pm 0.32$ & $0.94 \pm 0.31$ & $7.32 \pm 0.70$ \\
\hline & $\mathrm{FR}^{\mathrm{a}}$ & $1.49 \mid 0.50$ & $0.91 \mid 0.48$ & $0.34 \mid 0.12$ & $0.13 \mid 0.01$ & $2.86 \mid 1.09$ \\
\hline \multirow[t]{3}{*}{ Areal conc. $\left(\mathrm{g} \mathrm{m}^{-2}\right)$} & LR-replete & $0.78 \pm 0.19$ & $0.41 \pm 0.10$ & $0.26 \pm 0.10$ & $0.04 \pm 0.03$ & $1.48 \pm 0.24$ \\
\hline & LR-deplete & $0.97 \pm 0.22$ & $0.50 \pm 0.10$ & $0.66 \pm 0.14$ & $0.31 \pm 0.08$ & $2.44 \pm 0.38$ \\
\hline & $\mathrm{FR}^{\mathrm{a}}$ & $0.98 \mid 0.33$ & $0.60 \mid 0.31$ & $0.22 \mid 0.08$ & $0.09 \mid 0.01$ & $1.89 \mid 0.72$ \\
\hline
\end{tabular}

LR: Laboratory scale RABR.

FR: Field scale RABR.

a Mean and range (I) for $n=2$.

Table 3

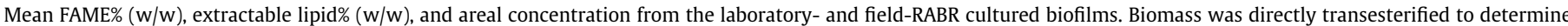

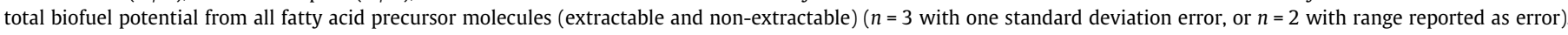

\begin{tabular}{|c|c|c|c|c|c|c|c|c|c|}
\hline \multirow[t]{2}{*}{ Fatty acids } & \multicolumn{3}{|l|}{ FAME\% } & \multicolumn{3}{|c|}{ Weight\% (w/w) } & \multicolumn{3}{|c|}{ Areal concentration $\left(\mathrm{g} \mathrm{m}^{-2}\right)$} \\
\hline & LR-replete & LR-deplete & $\mathrm{FR}^{\mathrm{a}}$ & LR-replete & LR-deplete & $\mathrm{FR}^{\mathrm{a}}$ & LR-replete & LR-deplete & $\mathrm{FR}^{\mathrm{a}}$ \\
\hline $\mathrm{C} 12: 0$ & $\mathrm{~N} / \mathrm{D}$ & $0.24 \pm 0.06$ & $0.54 \mid 0.04$ & $\mathrm{~N} / \mathrm{D}$ & $0.03 \pm 0.01$ & $0.03 \mid 0.01$ & $\mathrm{~N} / \mathrm{D}$ & $0.01 \pm 0.002$ & $0.02 \mid 0.005$ \\
\hline C14:0 & $0.39 \pm 0.01$ & $0.39 \pm 0.06$ & $1.65 \mid 0.69$ & $0.03 \pm 0.003$ & $0.04 \pm 0.01$ & $0.08 \mid 0.02$ & $0.01 \pm 0.002$ & $0.01 \pm 0.003$ & $0.05 \mid 0.01$ \\
\hline C15:0 & $0.40 \pm 0.02$ & $0.43 \pm 0.09$ & $0.60 \mid 0.04$ & $0.03 \pm 0.003$ & $0.05 \pm 0.01$ & $0.03 \mid 0.01$ & $0.01 \pm 0.002$ & $0.02 \pm 0.004$ & $0.02 \mid 0.01$ \\
\hline C16:1-3 & $19.85 \pm 1.34$ & $19.50 \pm 0.41$ & $22.97 \mid 2.64$ & $1.67 \pm 0.02$ & $2.05 \pm 0.03$ & $1.17 \mid 0.36$ & $0.58 \pm 0.05$ & $0.68 \pm 0.05$ & $0.77 \mid 0.24$ \\
\hline C16:0 & $16.68 \pm 1.61$ & $18.68 \pm 1.14$ & $15.58 \mid 0.93$ & $1.41 \pm 0.23$ & $1.96 \pm 0.06$ & $0.79 \mid 0.11$ & $0.49 \pm 0.11$ & $0.65 \pm 0.06$ & $0.52 \mid 0.07$ \\
\hline C18:1-3 & $50.15 \pm 2.60$ & $50.42 \pm 1.76$ & $42.28 \mid 2.02$ & $4.24 \pm 0.47$ & $5.30 \pm 0.34$ & $2.15 \mid 0.52$ & $1.47 \pm 0.27$ & $1.76 \pm 0.16$ & $1.42 \mid 0.34$ \\
\hline C18:0 & $0.88 \pm 0.08$ & $0.95 \pm 0.07$ & $2.90 \mid 3.21$ & $0.07 \pm 0.01$ & $0.10 \pm 0.01$ & $0.14 \mid 0.13$ & $0.03 \pm 0.01$ & $0.03 \pm 0.004$ & $0.09 \mid 0.09$ \\
\hline C20:4-5 & $3.35 \pm 1.12$ & $2.51 \pm 0.65$ & $5.67 \mid 0.83$ & $0.28 \pm 0.10$ & $0.26 \pm 0.06$ & $0.29 \mid 0.10$ & $0.10 \pm 0.04$ & $0.09 \pm 0.03$ & $0.19 \mid 0.06$ \\
\hline C20:1-3 & $0.71 \pm 0.13$ & $0.64 \pm 0.19$ & $\mathrm{~N} / \mathrm{D}$ & $0.06 \pm 0.01$ & $0.07 \pm 0.02$ & $\mathrm{~N} / \mathrm{D}$ & $0.02 \pm 0.01$ & $0.02 \pm 0.01$ & $\mathrm{~N} / \mathrm{D}$ \\
\hline C20:0 & $\mathrm{N} / \mathrm{D}$ & $\mathrm{N} / \mathrm{D}$ & $0.18 \mid 0.37$ & $\mathrm{~N} / \mathrm{D}$ & $\mathrm{N} / \mathrm{D}$ & $0.01 \mid 0.02$ & $\mathrm{~N} / \mathrm{D}$ & $\mathrm{N} / \mathrm{D}$ & $0.01 \mid 0.01$ \\
\hline $\mathrm{C} 22: 0$ & $0.48 \pm 0.08$ & $0.51 \pm 0.13$ & $0.63 \mid 0.07$ & $0.04 \pm 0.01$ & $0.05 \pm 0.01$ & $0.03 \mid 0.01$ & $0.01 \pm 0.004$ & $0.02 \pm 0.01$ & $0.02 \mid 0.01$ \\
\hline $\mathrm{C} 24: 0$ & $0.28 \pm 0.04$ & $0.29 \pm 0.01$ & $0.63 \mid 0.03$ & $0.02 \pm 0.002$ & $0.03 \pm 0.000$ & $0.03 \mid 0.005$ & $0.01 \pm 0.000$ & $0.01 \pm 0.001$ & $0.02 \mid 0.003$ \\
\hline $\mathrm{C} 26: 0$ & $0.37 \pm 0.03$ & $0.42 \pm 0.04$ & $0.56 \mid 0.07$ & $0.03 \pm 0.003$ & $0.04 \pm 0.004$ & $0.03 \mid 0.002$ & $0.01 \pm 0.002$ & $0.01 \pm 0.003$ & $0.02 \mid 0.001$ \\
\hline C28:0 & $\mathrm{N} / \mathrm{D}$ & $0.35 \pm 0.31$ & $0.90 \mid 0.41$ & $\mathrm{~N} / \mathrm{D}$ & $0.04 \pm 0.03$ & $0.04 \mid 0.01$ & $\mathrm{~N} / \mathrm{D}$ & $0.01 \pm 0.01$ & $0.03 \mid 0.01$ \\
\hline Other & $6.46 \pm 2.45$ & $4.68 \pm 1.30$ & $4.90 \mid 0.05$ & $0.54 \pm 0.18$ & $0.49 \pm 0.14$ & $0.25 \mid 0.05$ & $0.18 \pm 0.05$ & $0.16 \pm 0.04$ & $0.16 \mid 0.03$ \\
\hline Total & 100 & 100 & 100 & $8.45 \pm 0.61$ & $10.51 \pm 0.33$ & $5.07 \mid 0.99$ & $2.92 \pm 0.42$ & $3.49 \pm 0.28$ & $3.34 \mid 0.65$ \\
\hline
\end{tabular}

LR: laboratory scale RABR.

FR: field scale RABR

N/D: Not detected.

${ }^{\text {a }}$ Mean and range $(\mid)$ for $n=2$. 
evaluated as the portion of the biofilm between the surface and $L_{\text {phot }}$, were practically indistinguishable (within $25 \mu \mathrm{m}$ ) between the two nitrate availability conditions. This measurement supports the observation that actinic light was fully attenuated by the same depth and that the oxygenic photosynthesis reaction volumes were near identical under both conditions.

Differences in the net areal rates of photosynthesis (both $P_{n}$ and $P_{n, p h o t}$ ) between the two nitrate availability conditions were not as pronounced. However, both $P_{n}$ and $P_{n, p h o t}$ represented a greater percentage of $P_{g}$ under nitrate deplete conditions. This observation is attributed to lower areal rates of photosynthetically-coupled respiration during nitrate starvation. Again, the $R_{\text {light }}$ and $R_{\text {phot }}$ values were measured as the difference between $P_{g}$ and respective net areal photosynthesis rates. Nitrate replete conditions promoted approximately $20 \%$ increase in photosynthesis coupled respiration rates. The $R_{\text {dark }}$ measurements were greater during nitrate starvation indicating a higher capacity for heterotrophic (or light independent) respiration. However, the maximum areal respiration rates were observed during illumination and corresponded with increased $P_{\mathrm{g}}$. This was consistent with the observations made on the field-RABR biofilms.

Although as a whole, there were only small differences observed in rates of photosynthesis and respiration between the nitrogen replete and deplete lab-RABRs, the data suggest two important findings: First, nitrate depletion in the medium might not have a strong effect on the general physiology of the biofilm because only a small fraction of the biofilm (outer surface) is actively performing photochemical production even under nitrogen replete conditions; secondly, the biofilms remained photosynthetically active under non-growth conditions highlighting the importance of maintenance energy for cell viability and the potential for nitrogen (re-) cycling. These are important observations, within the setting of algal lipid production, since nitrogen stress is a common strategy for triggering triacylgycerol accumulation in planktonic microalgal cultures (Converti et al., 2009; Mus et al., 2013; Stephenson et al., 2010).

The first specific aim of this study was to characterize and compare the two different RABR biofilms (lab- and field-scale) in the context of active photosynthesis and spatial gradients in steadystate oxygen and photosynthesis. Of the physiological parameters measured for this specific aim, photosynthesis-coupled respiration is of special interest and should be considered a potent design parameter for controlling local $\mathrm{O}_{2} / \mathrm{CO}_{2}$ ratios to promote carbon fixation and subsequent photo-productivity. One potential strategy for maximizing gross photosynthesis while minimizing localized oxygen concentration would be to promote heterotrophic activity via mixed culturing techniques. Evidence for this lies in the observation that the field-RABR top-oriented biofilm community, as compared to the WC-2B lab-RABR biofilms, displayed a higher potential for electron acquisition from the environment (proportional to $P_{g}$ ) while channeling much greater percentages of photosynthetically derived oxygen into respiration processes. A combination of the wastewater remediating and biofuel production processes, may be better achieved via mixed species inoculation or 'seeding' with known lipid accumulating photoautotrophic community members combined with compatible heterotrophic oxygen scavengers. Consortial cooperation in microbial biofilm technology has previously been demonstrated in a number of different cell factory systems (Bernstein and Carlson, 2012).

\subsection{Biofuel precursor production}

Extractable lipid fractions were recovered from all biofilm samples and analyzed by gas chromatography for assessment of biofuel properties (Table 2). In addition, direct transesterification was performed on the lyophilized biomass to identify fatty acids and to determine total biofuel potential (extractable and non-extractable) for each biofilm-type (Table 3). Modest increases of extractable precursor concentrations were measured in the nitrate deplete biofilms, as compared to the nitrate replete conditions. This observation was also qualitatively confirmed in microscopy images (compare Fig. S2A and S2B); where Bodipy 505/515 was used to visualize the neutral lipid precursors. The total FAME-weight\%, representative of the total biofuel potential of the biofilm, was also modestly higher for the lab-RABR biofilms that were deplete of nitrate.

The most notable differences regarding lipid production in the lab-RABR biofilms, were the differences in the total extractable weight\% of lipids (sum of the FFA, MAG, DAG, and TAGs) between the nitrate replete and deplete conditions, $4.3 \pm 0.4 \%$ and $7.3 \pm 0.7 \%$ $(w / w)$, respectively (Table 2$)$. The largest differences were observed in the DAG and TAG weight\% and the respective areal concentrations. Although the WC-2B biofilms exhibited reasonable biofuel potentials; the lab-RABR production-system is not considered optimized for biofuel production. Only $7.3 \%(\mathrm{w} / \mathrm{w})$ of extractable precursors accumulated, which is significantly less than planktonic cultures of WC-2B that can accumulate up to $13.9 \%$ $(\mathrm{w} / \mathrm{w})$ of extractable precursors $(7.7 \%(\mathrm{w} / \mathrm{w})$ of which is TAG) under high $\mathrm{pH}$ and nitrate deplete conditions (Gardner, unpublished data). This evinces that medium nitrate depletion alone may not be an effective condition for inducing TAG accumulation in microalgal biofilms, likely due to heterogeneous distributions of nutrients like nitrate caused by mass transfer limitations as well as differences in $\mathrm{pH}$ stress and differences in inorganic carbon availability throughout the biofilms. It should be noted that comparisons of these preliminary biofilm oil-production systems to wellmixed planktonic systems does not account for culturing times, biomass production rates or differences associated with required operating costs (e.g., energy required for mixing, biomass harvesting or water input requirements).

Biofilms cultured on the field-RABR had the lowest weight percentage in both extractable precursor molecules and potential FAMEs, $2.9 \pm 1.1 \%$ and $5.1 \pm 1.0 \%(\mathrm{w} / \mathrm{w})$, respectively (Tables 2 and 3 ). This observation coincides with the relatively high respiration rates measured in the samples (discussed earlier). The fieldRABR biofilm samples are clearly not optimized for biodiesel production (i.e., total FAMEs) under the current culturing conditions. This could be, in part, due to colonization of a non-lipid accumulating microbial community native to the wastewater (Fig. S2). However, the field-RABR exhibited higher biomass productivity $\left(P=\Delta \mathrm{g}_{\mathrm{cdw}} / \Delta\right.$ time $)$ and total biomass areal density compared to the lab-RABR. Hence, the amount of FAME recoverable from the field-RABR biofilms per unit area was similar to the amount recoverable from the nitrate deplete WC-2B biofilms from the lab-RABRs (Table 3).

The second specific aim of this study was to characterize and compare the biofuel potential and (neutral lipid) precursor biomolecule composition in these biofilms. Although the current RABR systems are not considered optimized, lipid accumulation in algal biofilms is possible and reasonable if the microbial composition is constrained to known lipid producers such as the WC-2B isolate used here in the lab-RABR system. Future optimization is needed including the investigation of other industrially relevant algal strains such as Botryococcus braunii or Chlorella vulgaris. The field-RABR design is a more practical and industrially scalable system compared to the lab-RABR. However, the current system is not considered viable for biodiesel production since it only accumulated $2.9 \pm 1.1 \%$ and $5.1 \pm 1.0 \%(\mathrm{w} / \mathrm{w})$ precursor molecules and FAMEs, respectively. Future optimization and experimentation of the field-scale system will require methodologies for enhanced control of the microbial community composition to select for better lipid accumulation. It should be noted that although biodiesel 
production from fatty acid containing precursor compounds is low in the field system, it is a potentially viable technique for biomass production from wastewater. Biofuel production from the fieldRABR has previously been reported through the conversion of algal biomass into acetone, butanol, and ethanol by Clostridium saccharoperbutylacetonicum (Ellis et al., 2012).

As part of the objective from specific aim 2, nitrate starvation was investigated as a potential strategy for inducing lipid accumulation in the lab-scale RABR cultures. Although a modest increase in extractable precursors was observed, nitrogen stress as implemented here with $60 \mathrm{~h}$ cultivation in the absence of nitrogen was not identified to be as effective in "triggering" lipid accumulation in biofilms as previously reported for suspended cultures (Gardner et al., 2011; Mus et al., 2013; Gardner, unpublished data). This biofilm specific result is consistent with another recently reported study which focused on nutrient starvation (including nitrate) in cultures composed of the fresh water green alga Scenedesmus obliquus and the marine diatom Nitzschia palea (Schnurr et al., 2013). That study tested biofilm growth and lipid accumulation in algae cultured under relatively low shear in flat plate biofilm reactors and reported no significant changes in lipid concentration (\% dry weight) between nitrate replete and deplete conditions. This is in minor contrast to the results from the current study which observed an approximate $2-3 \% \mathrm{w} / \mathrm{w}$ increase after nitrate depletion. Additionally, the Schnurr et al. study reported significant and near complete biomass sloughing post nitrate depletion which was not observed to the same extent in the lab-RABR biofilms during the $60 \mathrm{~h}$ of nitrate starvation. This could be due to the different substratum materials (i.e., glass-plate compared to cotton cord) or localized shear-stress at the biofilm surfaces. The combined results between the current and previously reported study (Schnurr et al., 2013) indicate that inducing lipid accumulation via nutrient starvation in biofilms may be possible but future research is needed to identify strategies for inducing lipid accumulation in algal biofilms, such as nitrogen or pH stress, or chemical addition (Gardner et al., 2011, 2012; Guckert and Cooksey, 1990; Mus et al., 2013; Valenzuela et al., 2012).

\section{Conclusion}

This manuscript explores critical photosynthetic parameters related to the production of biofuel precursor molecules by algal biofilms cultured on rotating algal biofilm reactors (RABR). The lab-RABR systems exhibited moderate biofuel capabilities yet require process optimization. The wastewater remediating fieldRABR systems exhibited higher rates of photosynthesis and respiration depending on the position with respect to sunlight, but are not currently a viable biodiesel production platform. This study developed a methodological foundation and establishes a benchmark for directly measuring photosynthetic parameters fundamental to understanding and ultimately controlling the physiology of algal biofilms and to designing efficient, photosynthetic platforms for biofilm-based product generation.

\section{Acknowledgements}

The authors acknowledge funding support from multiple sources: (1) the National Science Foundation Integrative Graduate and Education Training (NSF-IGERT) (DGE 0654336) and NSFSustainable Energy Pathways program (CHE-1230632); (2) Church \& Dwight Co., Inc.; (3) Department of Energy, Genomic Science Program-Foundational Scientific Focus (Pacific Northwest National Laboratory subcontract 112443 to MSU), as well as, the Energy Efficiency and Renewable Energy (EERE) Biomass Program (DE-EE0005993); (4) The Laboratory Directed Research and
Development Program at Pacific Northwest National Laboratories partially supporting H.C.B through the Linus Pauling Distinguished Postdoctoral Fellowship program; (5) the Utah Science Technology and Research (USTAR) program (Scott Hinton, PI); (6) the Logan City Environmental Department Award (Control Number 080441); and (7) the Utah Water Research Laboratory (Award WA-1089) for project support to Utah State University. This work was also partially made possible by microscope facilities at the Montana State University Center for Biofilm Engineering, which was supported by funding obtained from the NSF-MRI Program and the M.J. Murdock Charitable Trust. The Environmental and Biofilm Mass Spectrometry Facility (EBMSF) at MSU funded through DURIP Contract Number: W911NF0510255 and the MSU Thermal Biology Institute from the NASA Exobiology Program Project NAG5-8807 is acknowledged. The microelectrode equipment was supported by the NIH COBRE Center for Analysis of Cellular Mechanisms and Systems Biology (NIH P20RR024237).

\section{Appendix A. Supplementary data}

Supplementary data associated with this article can be found, in the online version, at http://dx.doi.org/10.1016/j.biortech.2014. 01.001 .

\section{References}

Bernstein, H.C., Beam, J.P., Kozubal, M.A., Carlson, R.P., Inskeep, W.P., 2013. In situ analysis of oxygen consumption and diffusive transport in high-temperature acidic iron-oxide microbial mats. Environ. Microbiol. 15, 2360-2370.

Bernstein, H.C., Carlson, R.P., 2012. Microbial consortia engineering for cellular factories: in vitro to in silico systems. Comput. Struct. Biotechnol. J. 3, <http:// dx.doi.org/10.5936/csbj.201210017>.

Boelee, N.C., Temmink, H., Janssen, M., Buisman, C.J.N., Wijffels, R.H., 2012. Scenario analysis of nutrient removal from municipal wastewater by microalgal biofilms. Water 4, 460-473.

Chisti, Y., 2007. Biodiesel from microalgae. Biotechnol. Adv. 25, 294-306.

Christenson, L., Sims, R., 2011. Production and harvesting of microalgae for wastewater treatment, biofuels, and bioproducts. Biotechnol. Adv. 29, 686702 .

Christenson, L.B., Sims, R.C., 2012. Rotating algal biofilm reactor and spool harvester for wastewater treatment with biofuels by-products. Biotechnol. Bioeng. 109, 1674-1684.

Converti, A. Casazza, A.A., Ortiz, E.Y., Perego, P., Del Borghi, M., 2009. Effect of temperature and nitrogen concentration on the growth and lipid content of Nannochloropsis oculata and Chlorella vulgaris for biodiesel production. Chem. Eng. Process.: Process Intensif. 48, 1146-1151.

Ellis, J.T., Hengge, N.N., Sims, R.C., Miller, C.D., 2012. Acetone, butanol, and ethanol production from wastewater algae. Bioresour. Technol. 111, 491-495.

Eustance, E., Gardner, R.D., Moll, K.M., Menicucci, J., Gerlach, R., Peyton, B.M., 2013. Growth, nitrogen utilization and biodiesel potential for two Chlorophytes grown on ammonium, nitrate or urea. J. Appl. Phycol.. http://dx.doi.org/ 10.1007/s10811-013-0008-5.

Falkowski, P.G., Raven, J.A., 1997. Aquatic Photosynthesis, vol. 256. Blackwell Science, Malden, MA.

Farooq, W., Lee, Y.-C., Ryu, B.-G., Kim, B.-H., Kim, H.-S., Choi, Y.-E., Yang, J.-W., 2013 Two-stage cultivation of two Chlorella sp. strains by simultaneous treatment of brewery wastewater and maximizing lipid productivity. Bioresour. Technol. 132, 230-238.

Gardner, R., Peters, P., Peyton, B., Cooksey, K.E., 2011. Medium pH and nitrate concentration effects on accumulation of triacylglycerol in two members of the Chlorophyta. J. Appl. Phycol. 23, 1005-1016.

Gardner, R.D., Cooksey, K.E., Mus, F., Macur, R., Moll, K., Eustance, E., Carlson, R.P. Gerlach, R., Fields, M.W., Peyton, B.M., 2012. Use of sodium bicarbonate to stimulate triacylglycerol accumulation in the Chlorophyte Scenedesmus sp. and the diatom Phaeodactylum tricornutum. J. Appl. Phycol. 24, 1311-1320.

Gardner, R.D., Lohman, E., Gerlach, R., Cooksey, K.E., Peyton, B.M., 2013. Comparison of $\mathrm{CO}_{2}$ and bicarbonate as inorganic carbon sources for triacylglycerol and starch accumulation in Chlamydomonas reinhardtii. Biotechnol. Bioeng. 110, $87-$ 96.

Glud, R.N., Ramsing, N.B., Revsbech, N.P., 1992. Photosynthesis and photosynthesiscoupled respiration in natural biofilms quantified with oxygen microsensors. J. Phycol. 28, 51-60.

Guckert, J.B., Cooksey, K.E., 1990. Triglyceride accumulation and fatty acid profile changes in Chlorella (Chlorophyta) during high $\mathrm{pH}$-induced cell cycle inhibtion. J. Phycol. 26, 72-79.

Hall-Stoodley, L., Costerton, J.W., Stoodley, P., 2004. Bacterial biofilms: from the natural environment to infectious diseases. Nat. Rev. Microbiol. 2, 95-108. 
Hu, Q., Sommerfeld, M., Jarvis, E., Ghirardi, M., Posewitz, M., Seibert, M., Darzins, A. 2008. Microalgal triacylglycerols as feedstocks for biofuel production: perspectives and advances. Plant J. 54, 621-639.

Jensen, J., Revsbech, N.P., 1989. Photosynthesis and respiration of a diatom biofilm cultured in a new gradient growth chamber. FEMS Microbiol. Lett, 62, 29-38.

Jorgensen, B.B., Marais, D.J.D., 1990. The diffusive boundary layer of sediments: oxygen microgradients over a microbial mat. Limnol. Oceanogr. 35, 1343-1355.

Kliphuis, A.M., Martens, D.E., Janssen, M., Wijffels, R.H., 2011. Effect of $\mathrm{O}_{2}: \mathrm{CO}_{2}$ ratio on the primary metabolism of Chlamydomonas reinhardtii. Biotechnol. Bioeng. 108, 2390-2402.

Kuhl, M., Glud, R.N., Ploug, H., Ramsing, N.B., 1996. Microenvironmental control of photosynthesis and photosynthesis-coupled respiration in an epilithic cyanobacterial biofilm. J. Phycol. 32, 799-812.

Lassen, C., Glud, R.N., Ramsing, N.B., Revsbech, N.P., 1998. A method to improve the spatial resolution of photosynthetic rates obtained by oxygen microsensors. J. Phycol. 34, 89-93.

Lohman, E.J., Gardner, R.D., Halverson, L., Macur, R.E., Peyton, B.M., Gerlach, R., 2013 An efficient and scalable extraction and quantification method for algal derived biofuel. J. Microbiol. Methods 94, 235-244.

Mus, F., Toussaint, J.-P., Cooksey, K.E., Fields, M.W., Gerlach, R., Peyton, B.M., Carlson, R.P., 2013. Physiological and molecular analysis of carbon source supplementation and $\mathrm{pH}$ stress-induced lipid accumulation in the marine diatom Phaeodactylum tricornutum. Appl. Microbiol. Biotechnol. 97, 3625-3642.

Ordog, V., Stirk, W.A., Lenobel, R., Bancirova, M., Strnad, M., van Staden, J., Szigeti, J. Nemeth, L., 2004. Screening microalgae for some potentially useful agricultural and pharmaceutical secondary metabolites. J. Appl. Phycol. 16, 309-314.
Ozkan, A., Kinney, K., Katz, L., Berberoglu, H., 2012. Reduction of water and energy requirement of algae cultivation using an algae biofilm photobioreactor Bioresour. Technol. 114, 542-548.

Pokoo-Aikins, G., Nadim, A., El-Halwagi, M., Mahalec, V., 2010. Design and analysis of biodiesel production from algae grown through carbon sequestration. Clean Technol. Environ. Policy 12, 239-254.

Revsbech, N.P., 1989. An oxygen microsensor with a guard cathode. Limnol. Oceanogr. 34, 474-478.

Schnurr, P., Espie, G.S., Allen, G., 2013. Algae biofilm growth and the potential to stimulate lipid accumulation through nutrient starvation. Bioresour. Technol. 136, 337-344.

Senousy, H.H., Beakes, G.W., Hack, E., 2004. Phylogenetic placement of Botryococcus braunii (Trebouxiophyceae) and Botryococcus sudeticus isolate UTEX 2629 (Chlorophyceae). J. Phycol. 40, 412-423.

Stephenson, A.L., Dennis, J.S., Howe, C.J., Scott, S.A., Smith, A.G., 2010. Influence of nitrogen-limitation regime on the production by Chlorella vulgaris of lipids for biodiesel feedstocks. Biofuels 1, 47-58.

Stewart, P.S., 1998. A review of experimental measurements of effective diffusive permeabilities and effective diffusion coefficients in biofilms. Biotechnol. Bioeng. 59, 261-272.

Stewart, P.S., Franklin, M.J., 2008. Physiological heterogeneity in biofilms. Nat. Rev, Microbiol. 6, 199-210.

Valenzuela, J., Mazurie, A., Carlson, R.P., Gerlach, R., Cooksey, K.E., Peyton, B.M., Fields, M.W., 2012. Potential role of multiple carbon fixation pathways during lipid accumulation in Phaeodactylum tricornutum. Biotechnol. Biofuels $5,40$. 